\title{
Analyzing Target-Based Cryptocurrency Pump and Dump Schemes
}

\author{
JT Hamrick \\ jth563@utulsa.edu \\ The University of Tulsa \\ USA \\ Marie Vasek \\ m.vasek@ucl.ac.uk \\ University College London
}

UK

\author{
Farhang Rouhi \\ University of New Mexico \\ USA
}

\author{
Tyler Moore \\ tyler-moore@utulsa.edu \\ The University of Tulsa
}

USA

\author{
Arghya Mukherjee \\ arm3606@utulsa.edu \\ The University of Tulsa \\ USA
}

\author{
Neil Gandal \\ gandal@post.tau.ac.il \\ Tel Aviv University \\ Israel
}

\begin{abstract}
As the number of cryptocurrencies has exploded in recent years, so too has the fraud. One popular strategy is when actors promote coordinated purchases of coins in hopes of temporarily driving up prices. Prior work investigating such pump and dump schemes has focused on the immediate impact to prices following pump signals, which were largely interpreted as following the same strategy. The reality, as with most cybercrimes, is that the operators of the schemes try out a much more heterogeneous mix of tactics. From a population of 12252 pump signals observed between July 2017 and January 2019, we identify and examine 3683 so-called target-based pump signals that announce promoted coins alongside buy and sell targets, but without a coordinated purchase time. We develop a strategy to measure the success of target pumps over longer time horizons. We find that around half of these pumps reach at least one of their sell targets, and that reaching their peak price often takes days, as opposed to the seconds or minutes required in pumps studied previously. We also examine the various groups promoting coins and present evidence that groups try a variety of distinct strategies and experience varying success. We find that the most successful groups promote many coins and issue many pumps, but not for the same coins. As decentralized finance becomes more popular, a deeper understanding of price manipulation techniques like target pumps is needed to combat fraud.
\end{abstract}

\section{CCS CONCEPTS}

- Security and privacy $\rightarrow$ Economics of security and privacy; - Social and professional topics $\rightarrow$ Financial crime.

\section{KEYWORDS}

cryptocurrencies, pump and dump schemes, price manipulation, social networks, cybercrime measurement

Permission to make digital or hard copies of all or part of this work for personal or classroom use is granted without fee provided that copies are not made or distributed for profit or commercial advantage and that copies bear this notice and the full citation on the first page. Copyrights for components of this work owned by others than the author(s) must be honored. Abstracting with credit is permitted. To copy otherwise, or republish, to post on servers or to redistribute to lists, requires prior specific permission and/or a fee. Request permissions from permissions@acm.org.

DeFi '21, November 19, 2021, Virtual Event, Republic of Korea.

(c) 2021 Copyright held by the owner/author(s). Publication rights licensed to ACM. ACM ISBN 978-1-4503-8540-4/21/11 . \$15.00

https://doi.org/10.1145/3464967.3488591
ACM Reference Format:

JT Hamrick, Farhang Rouhi, Arghya Mukherjee, Marie Vasek, Tyler Moore, and Neil Gandal. 2021. Analyzing Target-Based Cryptocurrency Pump and Dump Schemes. In Proceedings of the 2021 ACM CCS Workshop on Decentralized Finance and Security (DeFi '21), November 19, 2021, Virtual Event, Republic of Korea. ACM, New York, NY, USA, 7 pages. https://doi.org/10. $1145 / 3464967.3488591$

\section{INTRODUCTION}

With thousands of cryptocurrencies circulating in an unregulated environment, it is not surprising that some traders try to manipulate prices for profit. One popular strategy is to pump coins, in hopes of driving up prices so that those behind the pump can liquidate their positions at the higher price. Pumpers cultivate communities on platforms such as Telegram and Discord, where members wait for announcements of the next coin to be pumped. These channels coordinate purchasing activities to push up prices. With names like "Whale Pump Group", traders understand that the purpose of the group is to push up prices in a way that members might profit.

Prior research has shown that the pumps can sometimes be spectacularly effective in the short term [8], even if most pumps are not particularly profitable [6]. Despite the fact that pump and dump signals vary greatly, prior work has treated them as the same during analysis. One strategy is to release information on a pumped coin incrementally, witholding the coin name until the precise moment traders are supposed to buy. Hamrick et al. termed such a strategy as a "countdown" pump. A contrasting strategy, called "target" pump, releases all information on the pumped coin at once. It also includes one or more price targets, and, optionally, stop loss values. Since prior analysis of cryptocurrency pump-and-dump schemes study the price change immediately surrounding the signal, they do not take into account the price targets mentioned in such pumps. The coins might take longer to reach the targeted values and prior work does not address whether target pumps are a successful strategy.

In this paper, we take a closer look at the various strategies employed by disparate pump groups. From a cybercrime measurement perspective, it is important to understand the different tactics employed by pump touts, as well as study how those strategies change over time. By increasing our understanding of the different strategies and their success, we can aid regulators and legitimate cryptocurrency operators in combating these illicit trading activities. Decentralized finance is likely to exacerbate the risk of such self-organized price manipulations, while making detection harder. 


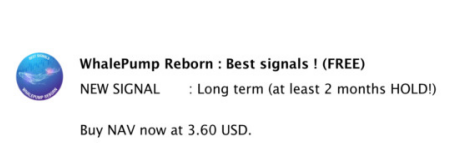

(a) Example pump signal from Whale Pump Group.

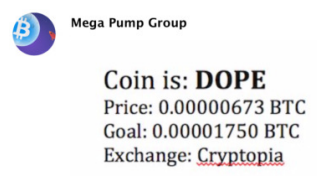

(b) Example pump signal from Mega Pump Group.

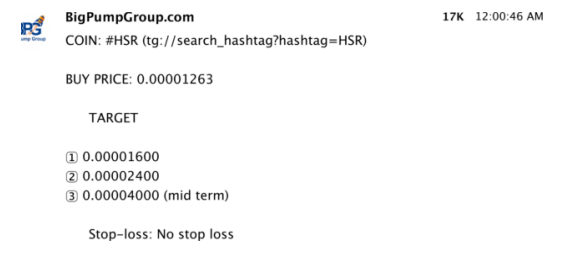

(c) Example pump signal from Big Pump Group.

Figure 1: Example target pump signals.

We make a number of contributions. Primarily, we systematically study target pumps. We extract information on price targets, describe their behavior, and construct novel methods to measure their success empirically. A second contribution is to study the behavior of the pump operators themselves. We examine the coins selected for promotion within groups and over time, uncovering widely varying strategies across groups.

\section{METHODOLOGY}

Hamrick et al. [5, 6] analyzed data on pumps between January and July 2018. The authors shared their data collection scripts with us, which we extended to gather pump signals from July 2018 through January 2019. Hence, our data collection methodology mirrors that discussed in [6]. Using active chat application accounts, pump signals were programmatically collected from the associated Telegram channels and Discord groups and then manually inspected them to verify whether the post was actually an attempted pump or not. We are confident that we obtained comprehensive data from the period we examine since we parsed all additional channels/groups that we discovered from those posts. This extended data contains 12252 signals of all types from 26 channels covering 294 cryptocurrencies.

We then set out to identify target signals, so we randomly selected signals and examined the message contents to identify differences in formatting. Figure 1 show examples of various target pump signals, which illustrates the differences in formatting used by promoters. Figure 1a only give buy targets which are more than likely the cryptocurrency's current price. Figure $1 \mathrm{~b}$ is an example of an incomplete target signal as it gives a buy and a sell target but nothing else. Complete target signals have a minimum of one value for each of the targets: stop-loss, buy, and sell. Traders could extrapolate missing values based on pump signal patterns. Nonetheless, in our analysis we restrict ourselves to explicitly stated values found in targets. The last signal in Figure 1c gives more information than the previous pump signals but would be considered an incomplete signal because of the lack of an explicit stop-loss target.

We took additional steps to identify and exclude non-target pumps. As the example posts show signal text formatting is rarely consistent between pump groups and can even be inconsistent within a single pump group. The process began with the development of robust regular expressions that would accurately locate target data within each of the pump signals. Once automated data parsing had completed, each of the pump signals was manually compared to the parsed data for correctness. A small number of missing or incorrect data was corrected during this manual inspection process.
We further pared down the data. We removed 3375 pump signals that did not specify a monetary target values. The collected pump signals contain a varying number of targets: 0-5 stop-loss values, 0-3 buy values, and 0-12 sell values. ${ }^{1}$ A further 1409 signals were removed since the cryptocurrencies were not tracked by coinmarketcap.com, so the corresponding pricing data could not be gathered. 1471 signals were removed as they were complete duplicates. Duplicates arise when the signals are transmitted across multiple groups, so we do consider them in Section 5.2 when we compare activities across groups. Clearing the dataset of unusable records left a total of 3683 meaningful distinct pump signals between July 2017 and January 2019 with at least one target value.

The analysis in following sections utilizes cryptocurrency price and volume data from coinmarketcap.com, which is the leading website for aggregate cryptocurrency trading data. We received information at 5-minute intervals, mapping 293 distinct cryptocurrencies to 3683 unique pump signals.

\section{IDENTIFYING TARGET-PUMP CYCLES AND SUCCESS}

Whereas prior work on cryptocurrency pump and dumps has focused on the immediate effect of pump signals, this is not necessarily the best way to measure success for signals using price targets. As explained in the previous section, target pumps typically do not coordinate traders by synchronizing signal timing. Instead, they bring traders together by setting common price targets. Consequently, in this section, we set out to describe an alternative measure of success that is based on the price targets set out in the pump signal.

\subsection{Pump Targets and Thresholds}

We first developed a method to flag points of interest within the cryptocurrency price data. These points of interest include the pump signal time as well as every occurrence of the cryptocurrency's price crossing a target value. Figure 2 gives an example of the method output. Horizontal lines indicate various price targets. The red dashed line indicates the pump signal time, while the purple dashed lines show when a threshold is crossed.

In the event that multiple targets exist for a pump signal, they are grouped by type and numbered. Figure 2 shows one stop-loss, two buy targets, and three sell targets for the Ark coin that was pumped on 2018-11-24. Moving through these targets from the bottom of

\footnotetext{
${ }^{1}$ It is worth noting that the $10^{\text {th }}$ through $12^{\text {th }}$ sell targets are never acquired for any of the pump signals for which they are explicitly given. The $9^{t h}$ target, which is more of a long-term target anyway, was only reached for one signal in the data and it was crossed roughly six months after the pump signal was posted.
} 


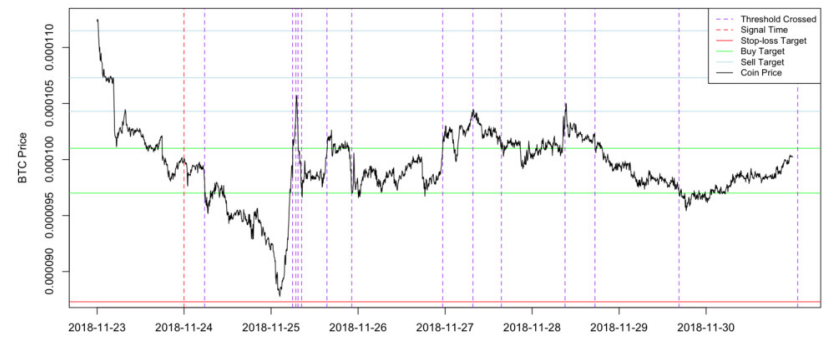

Figure 2: Ark pump activity from November 2018.

the figure to the top they are numbered as follows: stoploss1, buy1, buy2, sell1, sell2, and sell3.

Note that we only flag thresholds when first crossed after the signal or when a different threshold than the current one is crossed. The price can fluctuate below the next highest and above the next lowest target. So long as the price never crosses either target, it remains flagged at the current target. For example, if the buy 1 target is crossed, as it is in Figure 2 on 2018-11-24, it stays there until it crosses another target, which it does on 2018-11-25 despite nearly touching the stoploss1 target earlier that same day.

Overall, we see that $73 \%$ of the pump signals are immediately followed by the price crossing one of the buy targets, with $39 \%$ entering at buy $1,34 \%$ at buy 2 and just $0.05 \%$ entering at buy3. This is a good sign as it most likely means that these pump signals are not copied pumps. Conversely, the $1.5 \%$ of pump signals that are directly followed by the price achieving a sell target are suspected to be copied pumps. A further $9 \%$ the pump signals cross no thresholds for one of two reasons: the first being a gap in the data for that cryptocurrency ( $1.2 \%$ of signals), and the second being the prices simply never cross a defined target ( $8 \%$ of signals).

The median time gap between pump signal and crossing the first threshold is 1.3 hours. $75 \%$ of the complete pump signals reach a buy target within 14 hours. By contrast, 13\% of pump signals do not reach a buy target until at least 7 days after a pump signal. In these cases, the pumps could be more accurately considered to fail, since the success is achieved much later than the signal time.

\subsection{When are Target Pumps Successful?}

The following analysis treats pump signals as if they are actively being used to place buy and sell orders. We expect that participants observing the pump signal place price-contingent buy orders at the pump signal time (e.g., buy a coin if it is within the buy range, sell when it reaches one of the targets or drops to the stop-loss value). The targets provide the information needed to place orders for buying, selling, and setting a stop-loss.

Once posted by the pump group organizers, these target values never change. We leverage this fact to construct a method to observe the cycle of price fluctuations in a time-independent manner that corresponds to how traders view the pumps. We define a targetpump cycle as the time period starting when first crossing a buy target and concluding when either the stop-loss is crossed or trading stops (whichever comes first).

Within each cycle certain points of interest are identified. The top target crossed is flagged and the max price within that top zone

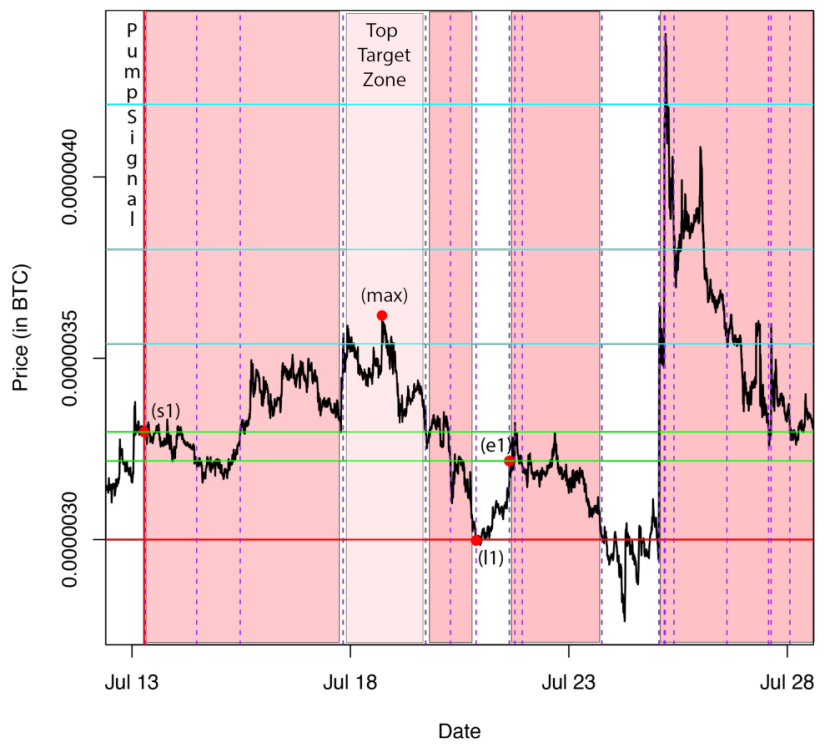

Figure 3: Cycle identification from cryptocurrency time series data (IOStoken).

is recorded. This top zone starts the first time the price crosses the top target and ends the last time the price crosses the target when it begins moving toward the subsequent stop-loss target. Within this zone the price could cross lower sell targets but for simplicity it will be treated as a "max zone." Finally, the lowest value is identified in the area between the max zone and the end of the current cycle. Figure 3 displays an example cycle and related points of interest.

A pump is successful if it reaches any sell target within this cycle, provided that it first started at a buy threshold or lower. The pump is unsuccessful if the price does not advance to a sell target. We interpret the most successful trading outcome for the pump organizers as the difference in price between the first buy target following the pump signal and the highest sell target achieved within the cycle. For unsuccessful pumps, we measure the difference in price between the first buy target in the cycle and the stop-loss price.

Because this method relies on the target values it can only use complete pump signals. Recall from Section 2 that pump signals contain varying levels of information and many are incomplete. To proceed, 2259 incomplete pump signals were removed. An additional 172 rows were removed because no signals were crossed (126), the pump signal target values were not in order (24), or no cycles were detected $(22)^{2}$. This leaves 1252 records with complete pump signal data. We refer to the broader dataset of 3683 signals as "target pump signals" and this narrower dataset of 1252 signals as "target cycle pump signals."

\section{ANALYZING TARGET PUMP CYCLES}

Having defined the pump cycle and success within it, we now investigate questions affecting all target cycle pumps.

\footnotetext{
${ }^{2}$ These pump signals began trading within the sell or stop-loss target zone and stayed within that zone. They never crossed a buy target.
} 
How Many Target Cycle Pumps Succeed? We determine pump success by looking for price movements across thresholds. Coins can experience multiple cycles (buy to stop-loss) after a pump signal. But should a target pump be deemed successful if it reaches a sell threshold after falling to the stop-loss level?

Table 1 considers three possible definitions. The most conservative only recognizes success if it is met during the first cycle following a pump signal. The most generous approach considers successful any pump where the sell target is met in a later cycle. Since many coins are pumped repeatedly, this is problematic. In between is to include all cycles until the next pump signal is reported.

\begin{tabular}{lrr}
\hline Measure & Successful & Unsuccessful \\
\hline First cycle only & 647 & 605 \\
Overall (cutoff at next signal) & 716 & 536 \\
Overall & 1002 & 250 \\
\hline
\end{tabular}

Table 1: Target pump success considering different cycles.

Table 1 reports the results. Around half of coins hit their sell target within the first cycle. If the time frame for success is extended beyond the first cycle many more pump signals are considered successful. The first measure of overall success ends the search window at the next pump signal for the coin being observed; if no future signals exist for a cryptocurrency then the cutoff date of the price data is used. There is an $11 \%$ increase in success between the first cycle and this first measure of overall success. If all subsequent pump signals are ignored and all trading cycles for a pump signal are grouped together then a 55\% increase in success can be observed when comparing to the first cycle group. This last measure of success is somewhat misleading because a handful of these pump signals have up to two years to experience success within a cycle.

The two overall measures are not necessarily realistic success measures when it comes to relating this analysis back to real-world trading activities. If traders lose out on the first cycle of the pump signal it is unlikely that they will submit the same trades and risk further losses. Hence, for subsequent analysis we define success by considering only the first cycle after the signal.

How Long Do Stages of the Target Pump Cycle Last? Figure 4 overlays summary statistics for the time elapsed between points of interest within a trading cycle onto pump price timelines. These summary values are grouped by pump success with an unsuccessful pump displayed on top and a successful pump on bottom. Median hourly values are reported (mean in parenthesis). The red rectangle simply displays the area in which the pump is in its "max zone."

Unsuccessful pumps fail faster than their successful counterparts succeed. Successful pumps take around 12 days to reach their maximum price target, compared to 8.8 hours for unsuccessful pumps. Successful coins spend 41 hours in the max zone, then remain 18 days before the cycle ends. Unsuccessful pumps stay in their peak target zone for 9.6 hours and conclude the cycle two days later.

Compared to countdown pumps, target pump price fluctuations operate on much longer timescales. This suggests that the overall pump phenomenon for target pumps is quite different to what has been considered in prior work, where the price jumps are measured on the scales of seconds and minutes rather than hours and days.

How Do Prices Rise and Fall During Pump Cycles? Since a stated goal of pump-and-dump activity is to increase coin prices, we now take a closer look at by how much the price actually moves. To quantify the magnitude of the price movements within a trading cycle, we calculated summary statistics for the points of interest outlined in Figure 3 (s1, max, 11).

\begin{tabular}{l|rr|rr}
\hline & \multicolumn{2}{|c}{ Successful } & \multicolumn{2}{c}{ Unsuccessful } \\
Range & Median & Mean & Median & Mean \\
\hline Start to max value & 29.6 & 54.2 & 2.7 & 5.3 \\
Max value to stop-loss & -51.9 & -4363.5 & -13.6 & -20.5 \\
\hline
\end{tabular}

Table 2: Percentage price movements between points of interest.

The figures are reported in Table 2. Successful pumps experience a $30 \%$ median price increase between the first target and the maximum price achieved during the pump cycle. The fall is even steeper: prices fall $52 \%$ (median) from the maximum price to the stop-loss value for successful pumps. Unsuccessful pumps see a much more modest median price increase of $2.7 \%$, though the decline is also smaller, falling $13.6 \%$ before hitting the stop-loss threshold.

\begin{tabular}{c|c|cc}
\hline $\begin{array}{c}\text { Days since } \\
\text { start }\end{array}$ & All & $\begin{array}{c}\text { \% Successful Pump } \\
\text { \& Hit Stop-loss }\end{array}$ & $\begin{array}{c}\text { \% Unsuccessful Pump } \\
\text { \& Hit Stop-loss }\end{array}$ \\
\hline 1 & $16.5 \%$ & $2.5 \%$ & $31.6 \%$ \\
2 & $23.6 \%$ & $4.5 \%$ & $44.0 \%$ \\
3 & $28.2 \%$ & $6.2 \%$ & $51.7 \%$ \\
4 & $32.9 \%$ & $8.3 \%$ & $59.2 \%$ \\
5 & $37.1 \%$ & $11.3 \%$ & $64.8 \%$ \\
6 & $40.3 \%$ & $13.0 \%$ & $69.4 \%$ \\
7 & $43.1 \%$ & $13.9 \%$ & $74.4 \%$ \\
\hline \# Signals & 1252 & 647 & 605 \\
\hline
\end{tabular}

Table 3: Stoploss crossed within $X$ days of cycle start (first cycle only).

Table 3 presents another way to look at the long-run price performance of pumped coins. It shows the percentage of pump signals that cross a stop-loss target between one and seven days following the pump signal. After one day only $16.5 \%$ of all pump signals have completed a cycle and crossed a stop-loss target. This rises to $33 \%$ after four days and finally $43 \%$ after one week.

Successful pumps are less likely to hit their stop targets quickly, with only $14 \%$ hitting a stop-loss target within 7 days. Unsuccessful pumps, on the other hand, reach stop-loss targets much faster. $32 \%$ cross a stop-loss target within the first 24 hours and just under $75 \%$ end their trading cycle within seven days of crossing the buy target.

\section{ANALYZING THE WIDER CONTEXT OF TARGET PUMPS}

We now take a closer look at the performance of target cycle pumps in the context of all target pumps observed, not just those with full cycle information as in the prior section. We also study how pump activity varies over time and across different groups. 

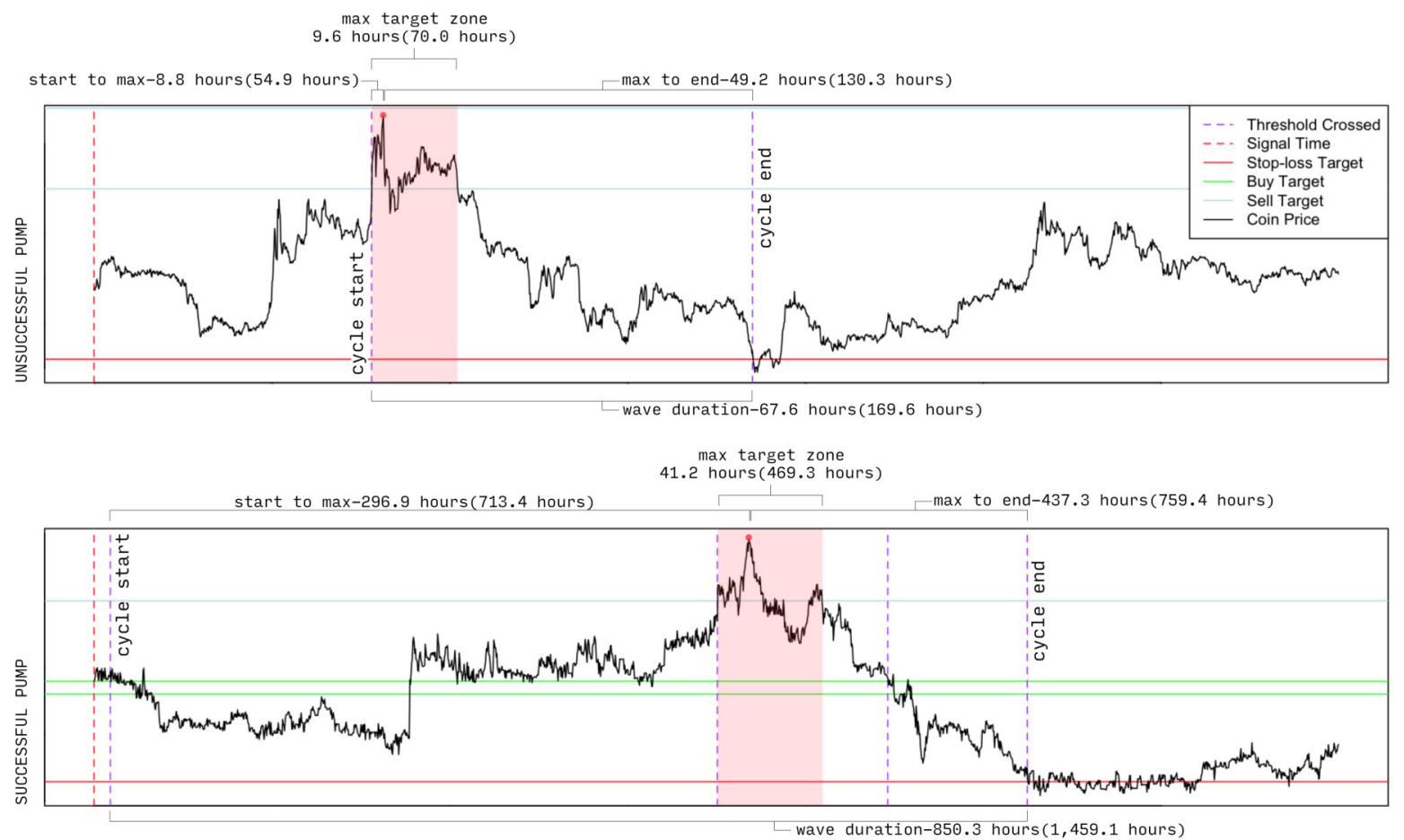

Figure 4: Cycle timing summary statistics - median (mean) hours.
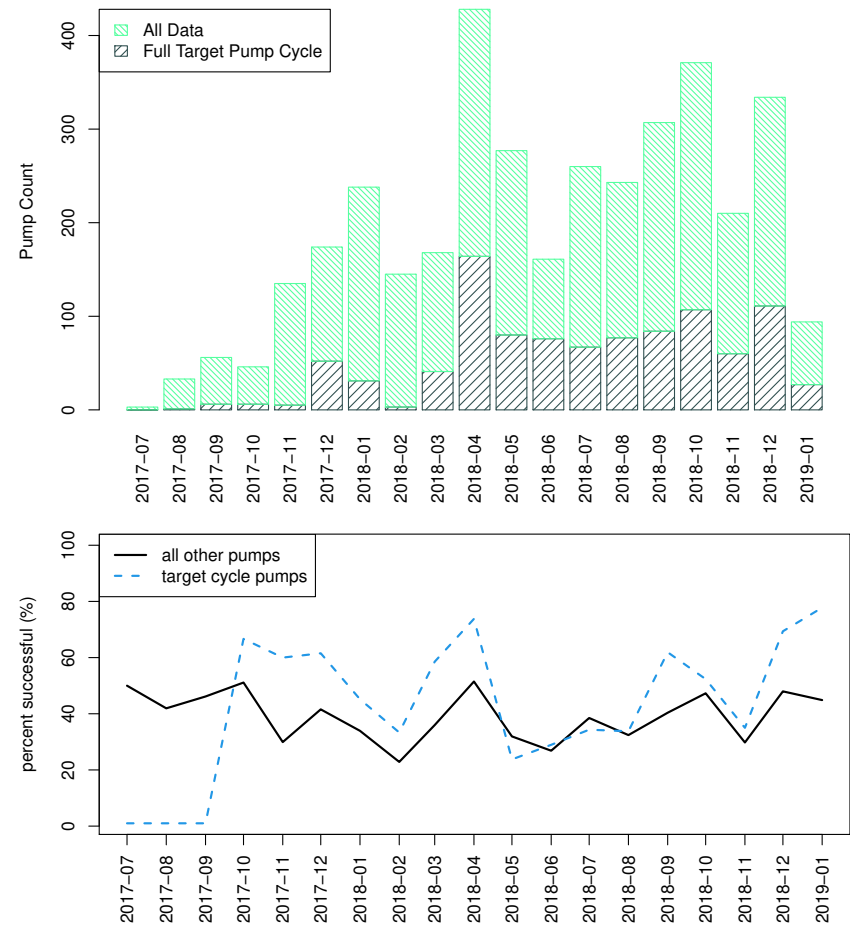

Figure 5: Frequency (top) and success (bottom) of pump signals over time, overall and for target cycle pumps.

\subsection{Target Pump Prevalence and Success Over Time}

Figure 5(a) plots the number of pump signals observed over time. The green bars indicate the number of target pumps seen overall, while the black shows the number of target pumps with complete buy, price and stop-loss figures (target cycle pumps). We can see that overall, pumps increased throughout 2017, peaking in April 2018. The number of pump signals varied from month to month but remained at a sustained high level. We can also see that the number of target cycle pumps rose from a very small number in 2017 to take up a much larger share of the total as time progressed.

Figure 5(b) plots the percentage of successful pumps, both overall and for target cycle pumps. Note that we adopted a different definition of success for the pumps lacking full target information. Here we measure whether they hit their sell target within an hour of the pump signal. While this is seemingly more aggressive than our cycle definition, it was fairly predictive of whether the first target cycle would be successful. The figure shows that success varies considerably from month to month. Once target cycle pumps become more prevalent in 2018, the success rate bounces between $25-75 \%$. This tends to outperform the success measure for other pumps, but we caution reading too much into that since the definitions of success are different. Finally, we note that the rate of success for all pumps does not appear to be consistently rising or declining. 


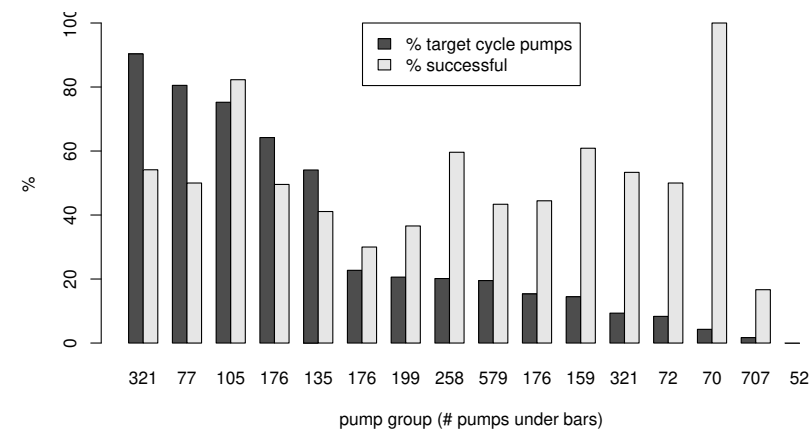

Figure 6: Target cycle pumps as a percentage of all pumps per group, with success rates. The total number of pumps per group is specified in the axis labels.

\subsection{Comparing Pump Group Strategies and Success}

Pump groups are independently maintained, and the operators decide which coins to pump and what form the signal message takes. We now take a closer look at how these groups differ. For this analysis, we exclude pump groups with fewer than 50 signals, bringing down the signals analyzed to 3583 across 16 groups.

Figure 6 examines the prevalence of target cycle pumps for the different groups. The dark bar indicates the percentage of pumps in the group that include full target cycles; the lighter color indicates the success rate of those pumps. The overall success rate for target cycle pumps is around $50 \%$. Finally, each group is labeled by the total number of pumps observed in that group. We can see that a few groups use predominantly cycle pumps, and their success is average. The exception is the third group, with 105 total pumps, which achieves an $80 \%$ success rate. Groups that do not specify buy, sell and stop-loss targets experience varying success.

Most groups pumped a wide variety of coins. 43 coins were only pumped by a single group. When these coins were pumped, they were only successful a third of the time, compared to the average pump success rate of $38 \% .^{3}$ However, coincidentally, another 43 coins were pumped by more than 10 groups. These coins account for 1256 pump signals. Associated pumps were more successful (40\%) than pumps involving less widely used coins (37\%). ${ }^{4}$ Moreover, we found that some groups repeatedly pumped the same coins within the group, while others did so much less often.

We now dig a bit deeper into these divergences in pumping strategy across groups. Two key metrics are the number of distinct coins pumped per day by the group and the number of pumps per day emanating from the group. Do groups succeed by issuing many pump signals? By targeting many different coins? By pumping a coin only once or by promoting the same coin repeatedly?

Figure 7 computes metrics for each group and relates them to each other and to pump success. Each point on the plot represents one of the 16 pump groups. Points are shaded based on the percentage of all pumps that succeed: red points indicate groups whose pumps usually succeed, while blue indicates groups that fail. The

\footnotetext{
${ }^{3}$ This difference is not statistically significant at the $5 \%$ level using a proportion test.

${ }^{4}$ This difference is statistically significant at the $5 \%$ level using a proportion test.
}

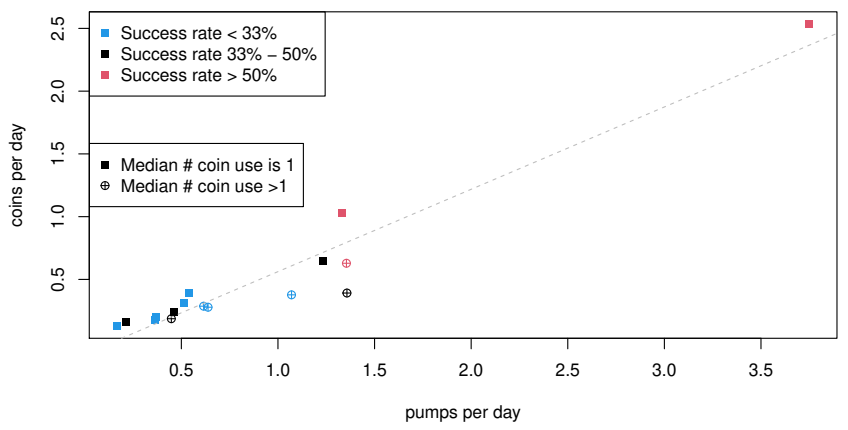

Figure 7: Comparing target pump success for among groups based on the frequency of sending pump signals and the variety of coins promoted.

$\mathrm{x}$-axis plots the number of target pump signals the group issues per day, while the $y$-axis indicates the number of distinct coins pumped per day. As expected, these metrics have a strong relationship. ${ }^{5}$ More surprising is that the groups that pump the most, in terms of distinct coins and number of signals, experience the most success. Groups that pump less often are less successful. Moreover, the most successful groups tend to not promote the same coins repeatedly.

\section{RELATED WORK}

There is a rich recent body of work analyzing the cryptocurrency pump and dump ecosystem [6-9, 11, 14]. Kamps and Kleinberg attempt to predict whether a coin is actively being used by one of these schemes [7]. They collected data across two pump and dump groups, and their work hinted at greater trends (like coin reuse) we analyzed further in this paper. Xu and Livshits refined the predictive work of Kamps and Kleinberg [14]. The basis of their model analyzed 412 pump and dump signals and referenced numerous trends (like pumps per day) that we studied further under the lens of target pumps. While their paper gave a case study of a countdown pump with building anticipation, much of their work on pump prediction could broadly apply to target pumps. The data provider for this paper, Hamrick et al., measured 3412 pump and dump schemes via Discord and Telegram over time and categorized each pump and dump scheme as "target" or "countdown" [6]. They found that less popular coins resulted in more successful pumps (higher price rises). They echoed similar results from $\mathrm{Xu}$ and Livshits finding concentrations in the ecosystem such as in exchanges and in highly popular Telegram and Discord channels. Notably, while Hamrick et al. did identify the existence of target pumps, they analyzed their impact on prices in the hours surrounding the pump signal. They also did not examine the price targets themselves. By contrast, we construct a time-independent method for analyzing price impacts relying on price target values reported in the signal. Closest to our work, Mirtaheri et al. also construct a definition of success based on target price, though their work does not explore this further [9].

Nonetheless, fraud in cryptocurrencies is not limited to this narrow form of market manipulation. Ponzi schemes, exit scams, manipulation by currency exchanges, and other forms of fraud are all rife in this ever evolving ecosystem $[1-4,10,12,13]$.

\footnotetext{
${ }^{5} \mathrm{~A}$ simple linear regression has an adjusted $R^{2}$ of 0.92 .
} 


\section{CONCLUDING REMARKS}

We have shed light on a pervasive yet underappreciated strategy used by pump-and-dump groups to temporarily drive up cryptocurrency prices. Target pumps attempt to coordinate traders so that they buy and sell at predetermined price bands, rather than all at once after the pump signal is transmitted. We extracted these targets from 3683 pump signals spanning more than eighteen months. We constructed a time-independent method for automatically identifying the pump cycle. We also constructed success criteria for target pumps, finding that around half "succeed" in reaching stated sell targets. However, in contrast to prior work on pumps that focused on immediate returns achieved within minutes, we have found that target pumps take far longer to achieve success (median 12 days).

We have also presented evidence that target pumps occupy a large and growing share of pump-and-dump activities on cryptocurrencies. This is significant because it should inform which countermeasures are most suitable. In some ways, these pumps should be easier for defenders to counter. Since target pumps take longer to drive up prices, detecting and blocking them within hours or even a few days could still make a big difference. In other respects, though, a shift towards target pumps could make policing them more difficult. We know that these signals represent pump-anddump activity because they are advertised in Telegram channels with names like "Big Pump Group". But what happens when the operators decide to rebrand as the crypto-equivalent of providing stock-picking advice? Setting price targets for coins could be construed as legitimate practice, even if its real aim is to coordinate purchases to deliberately drive up prices. As operator tactics continue to evolve, regulators must remain vigilant in monitoring and disrupting illicit activities.

\section{ACKNOWLEDGEMENTS}

We gratefully acknowledge support from the following research grants: US-Israel Binational Science Foundation Grant No. 2016622, US National Science Foundation Award No. 1714291, a Blavatnik
Interdisciplinary Cyber Research Center at Tel Aviv University grant, an Intel academic grant for basic research, and a grant from the Sapir Center for Development at Tel Aviv University.

\section{REFERENCES}

[1] Badawi, E., Jourdan, G.V., Bochmann, G., Onut, I.V.: An automatic detection and analysis of the Bitcoin generator scam. In: Proceedings of IEEE Security and Privacy on the Blockchain (2020)

[2] Bian, S., Deng, Z., Li, F., Monroe, W., Shi, P., Sun, Z., Wu, W., Wang, S., Wang, W.Y., Yuan, A., et al.: IcoRating: A deep-learning system for scam ICO identification (2018), available at: https://arxiv.org/pdf/1803.03670

[3] Gandal, N., Hamrick, J., Moore, T., Oberman, T.: Price manipulation in the bitcoin ecosystem. Journal of Monetary Economics 95, 86-96 (2018). https://doi.org/10.1016/j.jmoneco.2017.12.004

[4] Griffin, J.M., Shams, A.: Is bitcoin really untethered? The Journal of Finance 75(4), 1913-1964 (2020). https://doi.org/10.1111/jofi.12903, https://onlinelibrary.wiley. com/doi/abs/10.1111/jofi.12903

[5] Hamrick, J., Rouhi, F., Mukherjee, A., Feder, A., Gandal, N., Moore, T., Vasek, M., Gandal, N., Vasek, M.: The economics of cryptocurrency pump and dump schemes. In: 18th Workshop on the Economics of Information Security (WEIS) (2019)

[6] Hamrick, J., Rouhi, F., Mukherjee, A., Feder, A., Gandal, N., Moore, T., Vasek, M., Gandal, N., Vasek, M.: An examination of the cryptocurrency pump and dump ecosystem (2019), available at: https://papers.ssrn.com/sol3/papers.cfm?abstract_ id $=3303365$

[7] Kamps, J., Kleinberg, B.: To the moon: defining and detecting cryptocurrency pump-and-dumps. Crime Science 7(1), 18 (2018)

[8] Li, T., Shin, D., Wang, B.: Cryptocurrency pump-and-dump schemes (2018), available at: https://papers.ssrn.com/sol3/papers.cfm?abstract id=3267041.

[9] Mirtaheri, M., Abu-El-Haija, S., Morstatter, F., Steeg, G.V., Galstyan, A.: Identifying and analyzing cryptocurrency manipulations in social media. arXiv preprint arXiv:1902.03110 (2020)

[10] Moore, T., Christin, N.: Beware the middleman: Empirical analysis of Bitcoinexchange risk. In: Financial Cryptography and Data Security. Lecture Notes in Computer Science, vol. 7859, pp. 25-33. Springer (April 2013)

[11] Nizzoli, L., Tardelli, S., Avvenuti, M., Cresci, S., Tesconi, M., Ferrara, E.: Charting the landscape of online cryptocurrency manipulation. arXiv preprint arXiv:2001.10289 (2020)

[12] Soska, K., Christin, N.: Measuring the longitudinal evolution of the online anonymous marketplace ecosystem. In: 24th USENIX Security Symposium. pp. 33-48 (2015)

[13] Vasek, M., Moore, T.: There's no free lunch, even using Bitcoin: Tracking the popularity and profits of virtual currency scams. In: Financial Cryptography and Data Security. Lecture Notes in Computer Science, vol. 8975, pp. 44-61. Springer (January 2015)

[14] Xu, J., Livshits, B.: The anatomy of a cryptocurrency pump-and-dump scheme. In: 28th USENIX Security Symposium. pp. 1609-1625 (2019) 